\title{
Strain-Induced Crystallization, Part IV: Induction Time Analysis
}

\author{
G. S. Y. YEH, K. Z. HONG, and D. L. KRUEGER \\ Departments of Chemical Engineering and Materials \& Metallurgical Engineering \\ and Macromolecular Research Center \\ University of Michigan \\ Ann Arbor, Michigan 48109
}

\begin{abstract}
The empirical equation, $1 / t_{i}=A e^{E_{i} / R T}$, which expresses the exponential dependence of the reciprocal of crystallization induction time, $t_{i}$, has been analyzed and shown to be equivalent to the nucleation rate equations derived earlier in Part III (1). Consequently we have used the $t_{i}$ measurements obtained earlier by Krueger and Yeh to calculate not only the nucleation rate enhancements but also the melting point elevations, the relative crystal thickness changes and molecular coil extension ratios of shear-crystallization polyethylene. It is shown that polyethylene when crystallized between 129 and $131^{\circ} \mathrm{C}$ at shear rates between 1.56 and $9.70 \mathrm{sec}^{-1}$ can have melting point increases of 4.2 to $7.2^{\circ} \mathrm{C}$ and crystal thickness decreases of 20 to 25 percent, when compared to those crystallized at $130^{\circ} \mathrm{C}$ in the quiescent state. The predicted "coil" extension in the melt just prior to shear-induced crystallization ranges between 21 and 36 percent. The results of these analyses as well as those on nucleation rates of polyethylene oxide are discussed in detail.
\end{abstract}

\section{INTRODUCTION}

$I^{\mathrm{n}}$ n Part III (1) a nucleation theory for strain-induced crystallization (SIC) was formulated to show how nucleation rate, critical nucleus size and equilibrium melting temperature of polymers depend on the "structure" state in terms of melt entropy reductions, $\Delta S^{\prime}$. In Part IV, the nucleation rates of shear-crystallized polyethylene (PE), which can be shown to be readily obtainable from induction times, $t_{i}$, are used to predict corresponding changes in crystal thickness, equilibrium melting temperature and molecular coil extension ratio of the oriented melt just prior to SIC.

\section{INDUCTION TIME ANALYSIS}

The induction time data for shear-crystallized PE used in the present analysis were the same reported previously by Krueger and Yeh $(2,4)$ and Tan and Gogos (3). The induction time, defined by Krueger and Yeh as the time elapsed from the start of shear until a stress rise or a volume decrease is observed, is shown in Fig. 1 . They noted two very interesting relations in their studies, one relating an "induction shear strain," $\epsilon_{i}=t_{i} \dot{\gamma}$ $=A^{\prime} e^{-E_{i} / R T}$, to crystallization temperature, $T$, and the other relating the reciprocal of induction time to $\gamma$ and $T$ as follows:

$$
1 / t_{i}=A e^{E_{i} / R T}
$$

where $A=\gamma / A^{\prime}$ and $R$ is the gas constant. The two equations are therefore equivalent. $\epsilon_{i}$ has been shown (2) to be constant for a given crystallization temperature, but $t_{i}$ depends greatly on $\dot{\gamma}$ as well as on $T$. Equation 1 was found (4) also to be valid for quiescent crystallization (QC) as well. The pre-exponential frequency factor $A$ and the temperature coefficient $E_{i}$ for both QC and SIC of $\mathrm{PE}$ are included in Fig. 2. The value of $E_{i}$ is much smaller for SIC than QC and it remains a constant in the shear rates investigated. However, $A$ was found to be proportional to shear rate. Since the rate of QC is almost negligible (see Fig. 2) as compared to that of SIC, one can write the following general expression for both types of crystallization:

$$
1 / t_{i}=\left(A_{0} e^{E_{0} / R T}+f \dot{\gamma}\right) e^{E / R T}
$$

where $A_{o}$ is now the frequency factor in QC, $f$ is a proportionality constant, $\left(E_{o}+E\right)$ and $E$ are respectively $E_{i}$ for QC and SIC. Since $A_{0} e^{\left(E_{0} / R T\right)}$ is generally small compared to $f \dot{\gamma}, E q 2$ can be reduced to $E q 1$ at $\dot{\gamma}>0$.

\section{MEANING OF $1 / t_{i}$}

If we consider the induction time, $t_{i}$, obtained in our shear-crystallization experiments, as the time required to generate the same critical number of SIC nuclei per unit volume, $n^{*}$, required to cause an observable increase in shear stress, then $n * / t_{i}$ becomes the SIC nucleation rate, or

$$
N^{o}=\frac{n^{*}}{t_{i}}=n^{*} A e^{E_{i} / R T} \cong n^{*}\left(A_{0} e^{E_{0} / R T}+f \dot{\gamma}\right) e^{E / R T}
$$

Equation 3 can now be compared to another empirical nucleation rate equation, $N^{o}=a+b \dot{\gamma}$, obtained by 


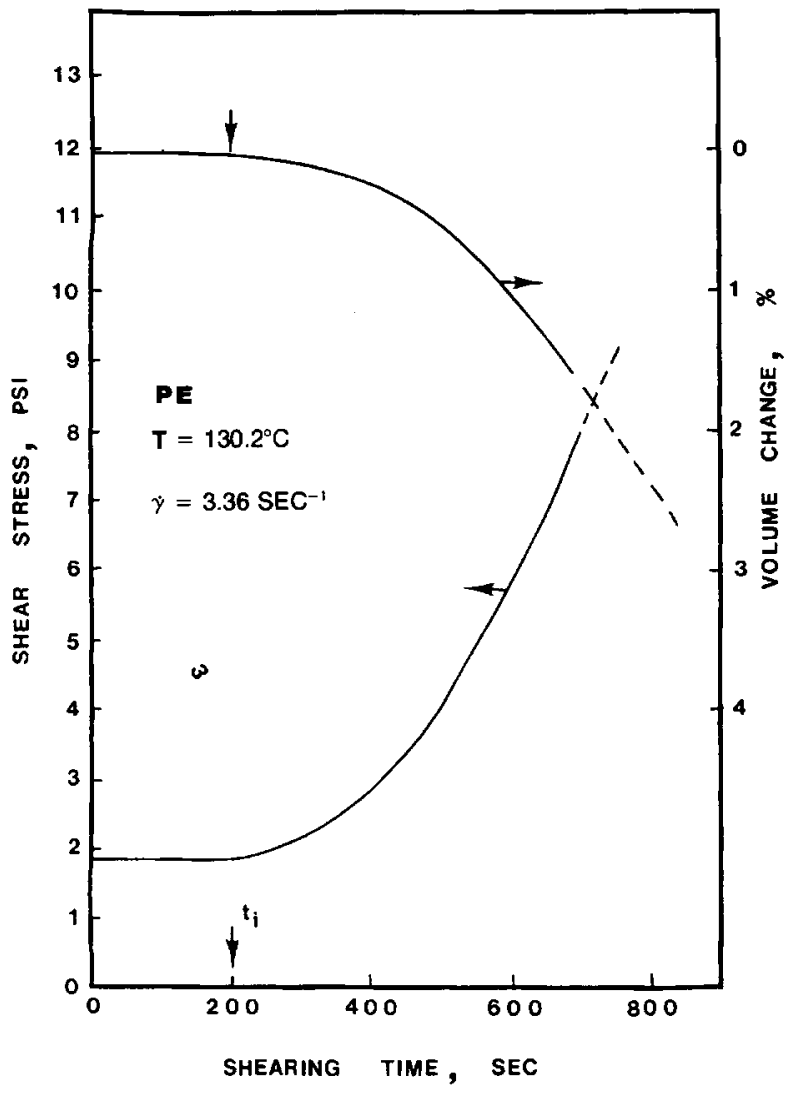

Fig. 1. Induction time, $t_{i}$, as deduced from stress or volume changes $(2,4)$.

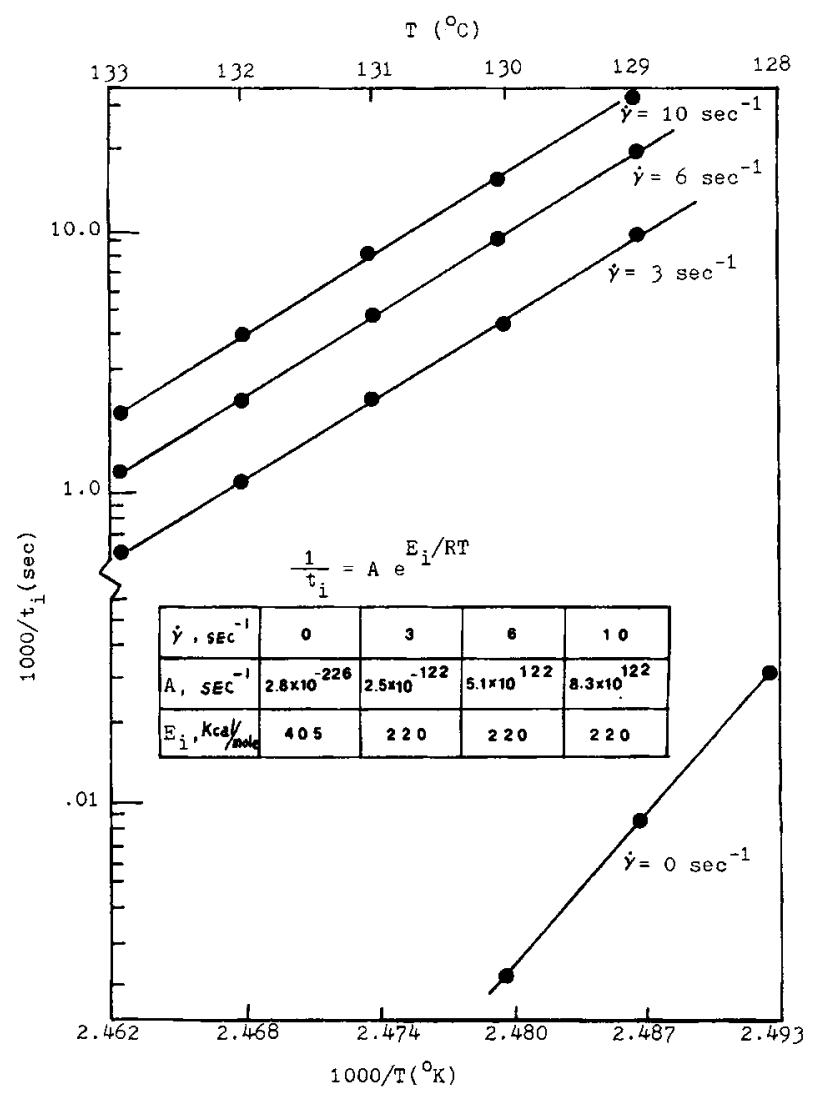

Fig. 2. Effects of crystallization temperature and shear rate on the reciprocal of induction time.
Ulrich and Price (5) who had formulated their equation to describe their observed nucleation rates of polyethylene oxide (by means of light microscopy) under various $\gamma$ and $T$. They had also found that the constant $a$ is negligible compared to $b$ and $b$ increases with decreasing $T$. This suggests that $a$ must correspond to $n * A_{o} \exp \left(E_{o}+\right.$ $E) / R T$, which as we pointed out earlier, is also negligible and $b$ to $n * f \exp (E / R T)$ in the more general nucleation rate expression $(E q 2)$ for polymers.

If we now compare the empirical nucleation rate expression, $E q 3$, with the general SIC nucleation rate expression (Part III (1)) namely

$$
N^{o}=N_{c} e^{-E_{D} / R T} e^{-\Delta F * o} / R T
$$

we can also show that they are equivalent to each other because $E q 4$ can be rewritten as

$$
N^{0}=N_{c} e^{(B-E D) / R T} e^{-(B+\Delta F * 0) / R T}
$$

If the constant $B$ is chosen such that $B-E_{D}=E$, then $E q 4^{\prime}$ becomes

$$
N^{0}=N_{c} e^{-(B+\Delta F * 0) / R T} e^{E / R T}
$$

Comparing Eqs 3 and 5, we find

$$
N_{c} e^{-(B+\Delta F * a) / R T}=n * f \dot{\gamma}
$$

This suggests that at constant temperature,

$$
\Delta F^{* o} \propto \ln (1 / \dot{\gamma})
$$

The relationships between the nucleation rate, $E q 3$, Eq 4 for SIC and the usual expression for quiescent crystallization are schematically shown in Fig. 3. Although $E q 3$ is an approximation to the theoretical expression $(E q 4)$, it has the advantage of having a simpler and more practical expression for describing the SIC behavior in terms of readily obtainable experimental parameters, namely, $\dot{\gamma}$ or stress, and $t_{i}$. However, it is valid only at high temperatures in the nucleationcontrolled regions where most of the SIC experiments are conducted anyway.

\section{ORIGIN OF RATE ENHANCEMENT}

The substantial increase in the front factor $A$ (see Fig. 2 ) in the case of SIC can now be explained based on the analyses made above. The increase is primarily due to the orientation effect on $\Delta F^{* 0}$. As discussed in Part III, the increase in the thermodynamic driving force, $\Delta f^{\circ}$,

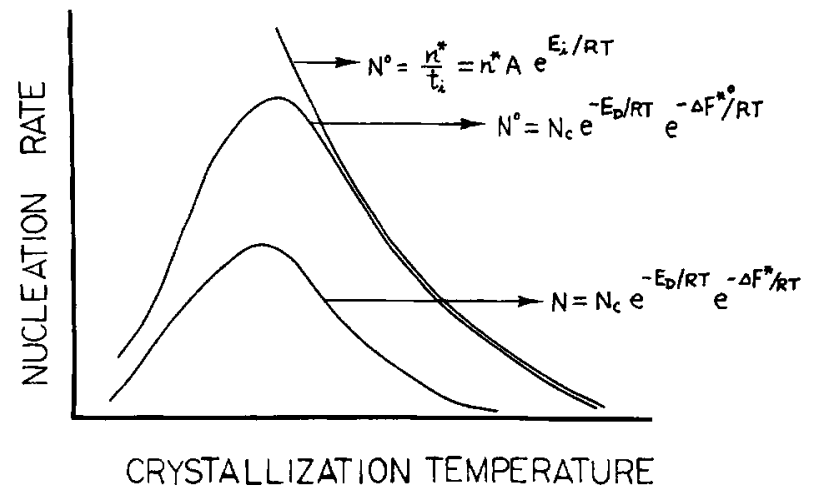

Fig. 3. Schematics showing the dependence of nucleation rate on temperature for the three indicated equations. 
brought about by melt orientation, results in a decreased $\Delta F^{* o}$ for the formation of a critical size nucleus of dimensions $\nu a^{* o}$ and $l^{* o}$. According to $E q 7$, the decrease in $\Delta F^{* 0}$ is clearly brought about by an increase in strain rate or stress imposed on the system during SIC, as was discussed in Part III.

The front factor, $A$, can be regarded as a measure of number of embryos participating in the nucleation process. Consequently the imposed strain on polymer melts has a dual effect on the enhancement of nucleation rate, by reducing the critical size nucleus to a smaller value (e.g., $\nu^{* 0}<\nu^{*}$ ) and by lowering the critical free energy $\Delta F^{* 0}\left(\left\langle\Delta F^{*}\right)\right.$. Both effects can lead to substantial enhancement of SIC nucleation rates $\left(N^{0}>>N\right)$, as schematically shown in Fig. 4 . The decrease in critical nucleus size is thermodynamically favorable for the increase of number of embryos larger than the critical size, whereas the lowering of free energy barrier, $\Delta F^{* \theta}$, is kinetically favorable for the formation of nuclei.

\section{PREDICTIONS OF $\mathrm{T}_{\mathrm{m}}^{\mathrm{o}}, l^{* o}$ AND $\alpha$ FROM $\mathrm{t}_{\mathrm{i}}$ DATA}

Based on the above analysis of the induction time equation $(E q 1)$, SIC nucleation rate enhancements were calculated for shear-crystallized polyethylenes. The results, together with corresponding melt entropy reductions $\left(\Delta S^{\prime}\right)$ and predicted values for $T_{m}^{0}, l^{* 0}$ and $\alpha$ using previously derived equations (see Table 1 ), are presented in Table 2 . Here $T_{m}^{o}$ is the equilibrium melting temperature for SIC polymer. In the analysis we have to assume that the SIC and the QC nucleation processes are both heterogeneous. If the nucleation processes were assumed to be both homogeneous, the

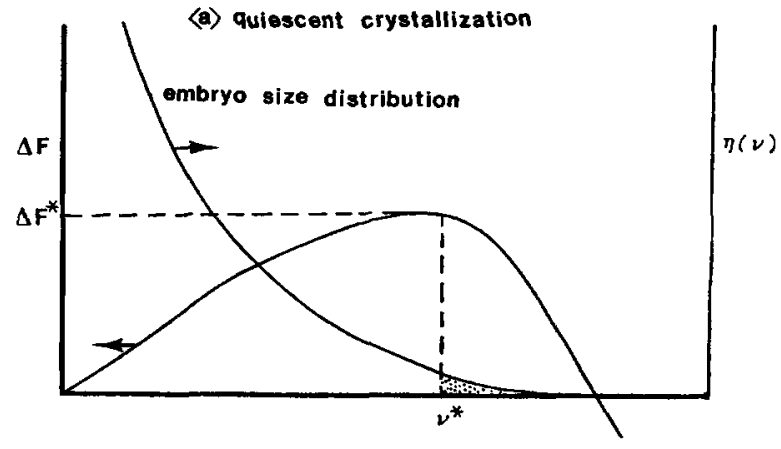

〈b〉strain-induced crystallization

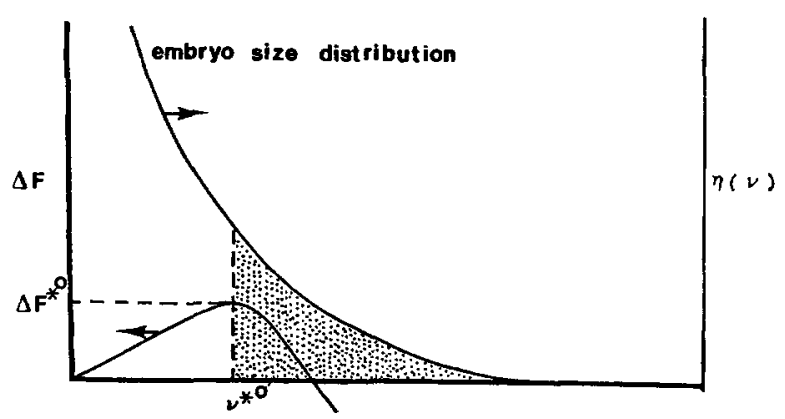

Fig. 4. Schematics showing the increase of matured embryos (shaded areas) upon reductions of critical size nucleus and critical free energy. $\eta(\nu)$ is the embryo size distribution.

predicted increase in $T_{m}^{o}$ would be at most only about $1^{\circ} \mathrm{C}$ above the isotropic equilibrium melting temperature, $T_{m}$, far smaller than the observed $5-7^{\circ} \mathrm{C}$ melting point increase recently obtained by Hong (6).

Table 1. Effects of Orientation, $\Delta \mathbf{S}^{\prime}(1)$

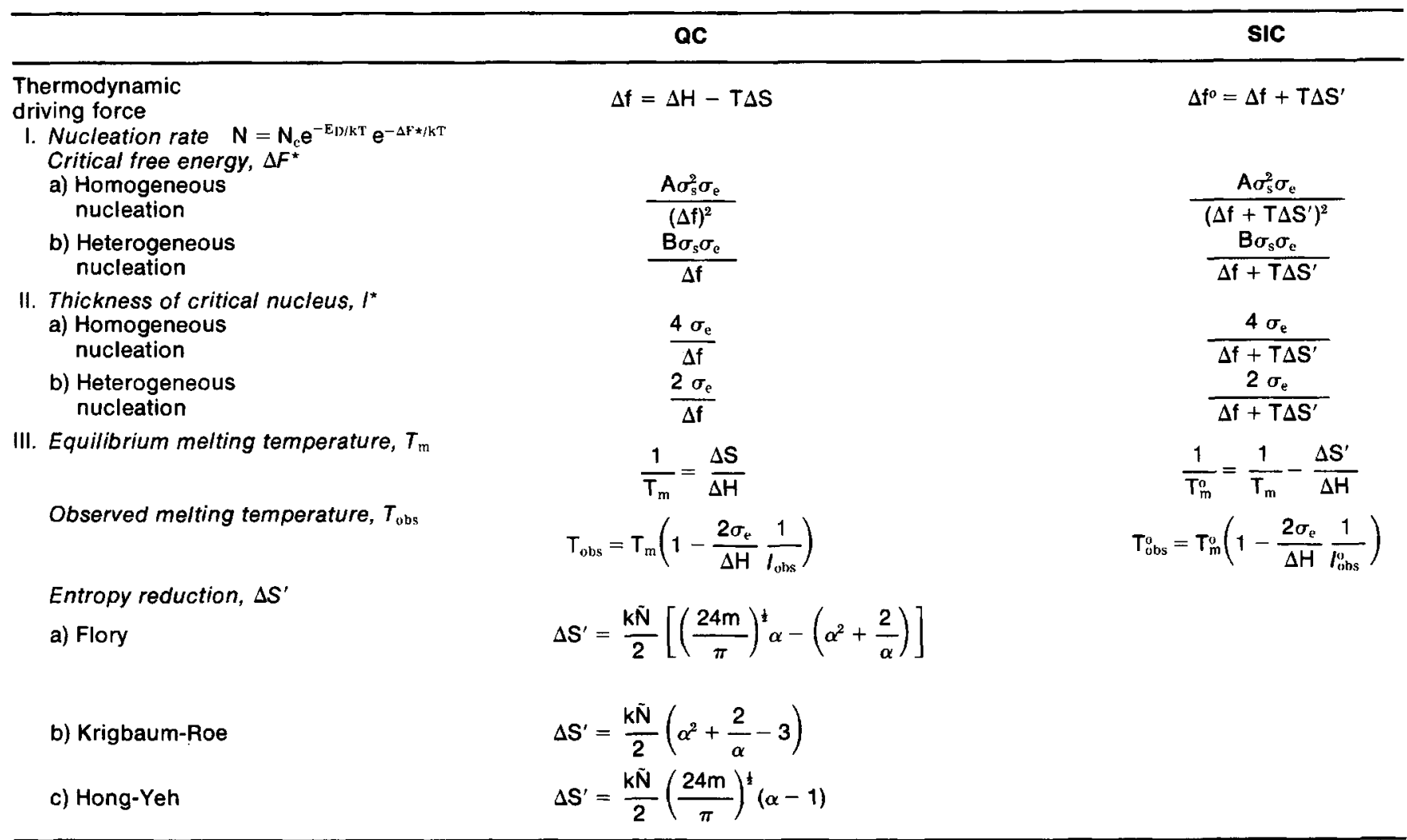


Table 2. Shear-Crystallized Polyethylene, Assuming Heterogeneous Nucleation



Constants used: $T_{m}=145^{\circ} \mathrm{C}, \sigma_{\mathrm{e}}=80 \mathrm{erg} / \mathrm{cm}^{2}, \sigma_{\mathrm{s}}=10 \mathrm{erg} / \mathrm{cm}^{2}, \Delta \mathrm{h}_{\mathrm{f}}=2.8 \times 10^{\circ} \mathrm{erg} / \mathrm{cm}^{3}, b_{\mathrm{b}}=4.11 \AA$ and $\tilde{\mathbf{N}}=1.5 \times 10^{20} / \mathrm{cm}^{3}$.

Further, a small increase of $1^{\circ} \mathrm{C}$ in $T_{m}$ will also lead to anomalies in the usual nucleation rate analyses (Figs. $5-7$, to be discussed later), thus giving another justification to the heterogeneous nucleation assumption used in the present analysis. The predicted decrease in $l^{* 0}$ of about 25 percent in Table 2 should, according to our SIC theory, reflect a corresponding 25 percent decrease in observed crystal thickness, $l_{o b s}^{o}$, or long period, $L_{o b s}^{o}$. A decrease of about 15 percent in $L_{o b s}^{o}$ from small-angle $\mathrm{X}$-ray measurements has indeed been noted earlier by Krueger (4) for these same specimens. However, the measured value of crystal thickness, $l_{o b s}^{o}$, is found to be
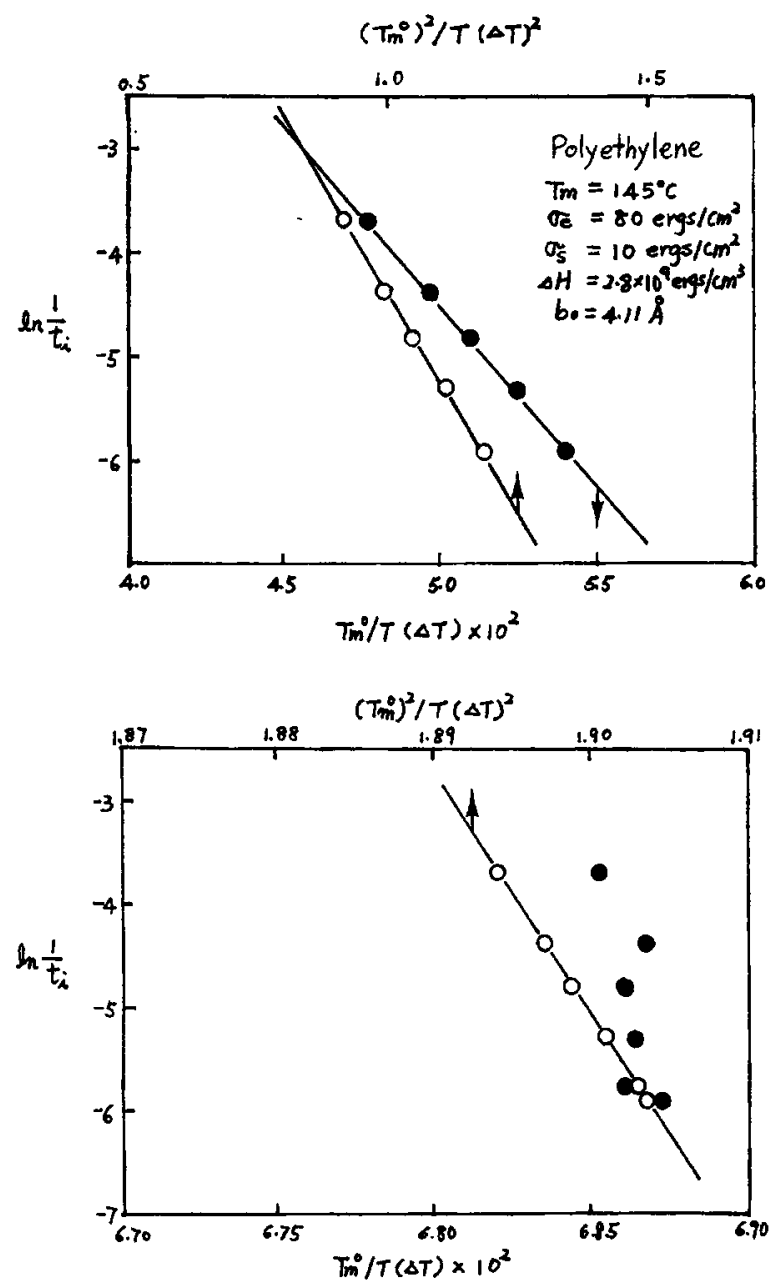

Fig. 5. Plot of $\ln 1 / t_{i}$ against $T_{m}^{o} /(\Delta T)$ and against $\left(T_{m}^{o}\right)^{2} / T(\Delta T)^{2}$ for polyethylene, using $t_{i}$ data provided by Krueger and $Y e h(2)$, and assuming heterogeneous nucleation (top) and homogeneous nucleation (bottom). about twice that of the theoretically predicted values of $l^{* o}$ shown in Table 2 . This, we believe, is due primarily to the omission of edge free energy and therefore the $\delta l$ term in our original derivation of $l * 0$.

It is again of interest to note that the "coil" extension ratio, $\alpha$, is only of the order of 30 percent, indicating that the molecules are only very slightly extended just prior to SIC for these specimens.
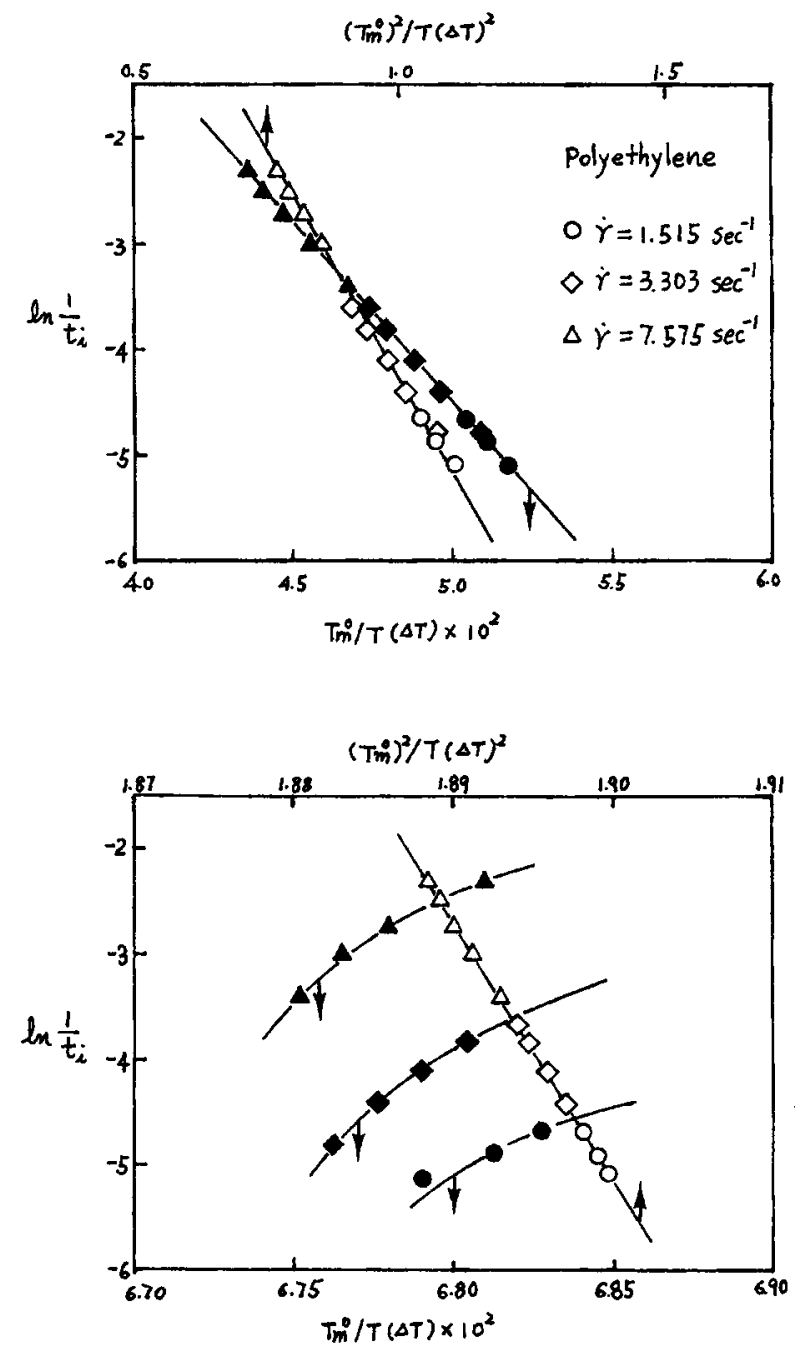

Fig. 6. Plot of $\ln 1 / t_{i}$ against $T_{m}^{o} / T(\Delta T)$ and against $\left(T_{m}^{o}\right)^{2} / T(\Delta T)^{2}$ for polyethylene, using $t_{i}$ data provided by Tan and Gogos (3), and assuming heterogeneous nucleation (top) and homogeneous nucleation (bottom). The constants used are the same as given in Fig. 5. 

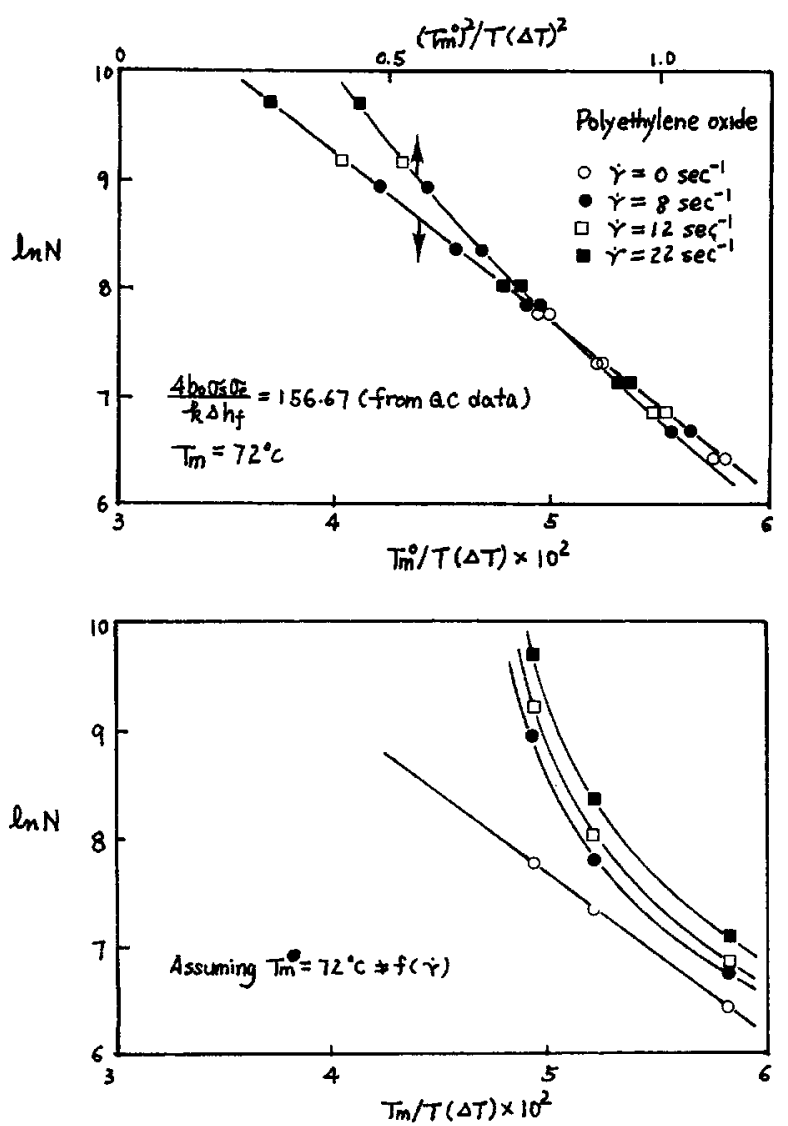

Fig. 7.Plot of In $N$ against $T_{m}^{o} / T(\Delta T)$ and against $\left(T_{m}^{o}\right)^{2} / T(\Delta T)^{2}$ for polyethylene oxide, using nucleation rate data provided by $\mathrm{Ul}$ rich and Price (5) and assuming heterogeneous nucleation (top and bottom). In the bottom figure when $T_{m}$ is assumed to be constant according to Ref. (5), the plots result in a series of curves for the shear cases.

\section{DISCUSSION ON PREDICTED $\mathrm{T}_{\mathrm{m}}^{\circ}$ VALUES}

Successful predictions of $T_{m}^{0}$ depend not only on reliable $N^{o} / N$ ratios to get $\Delta S^{\prime}$ values but also on the use of the Hong-Yeh $T_{m}^{o}$ equation (see Table 1) the validity of which will be discussed in Part V (8). For the present it would be desirable to test the accuracy of the predicted $T_{m}^{o}$ values using the classical tests by plotting $\ln$ (nucleation rate) vs $T_{m}^{o} / T(\Delta T)$ and $T_{m}^{o 2} / T(\Delta T)^{2}$. It has been shown that, at least in the case of QC, if the predicted $T_{m}^{a}$ values are correct, then such plots should lead to straight lines (9). We have applied similar tests to our data (Fig. 5), to the induction time data by Tan and Gogos (3) also on shear-crystallized PE (Fig. 6), and to the nucleation rate data by Ulrich and Price on polyethylene oxide (5) (Fig. 7). The $T_{m}^{o}$ values used in the lower plots of Figs. 5 and 6 are obtained from $\Delta S^{\prime}$ which are calculated from kinetic data according to the homogeneous nucleation rate equation, whereas the values of $T_{m}^{o}$ used in the upper plots of Figs. 5-7 are calculated following the heterogeneous nucleation rate equation (see Part III). In each case, if correctly predicted $T_{m}^{o}$ values are used in the plots, not only a straight line is obtained for the assumed crystallization process, e.g., heterogeneous (see Figs. 5-7), it can also be obtained in the ln (nucleation rate) vs $T_{m}^{o 2} / T(\Delta T)^{2}$ plots. Otherwise, incorrect assumptions of crystallization process and/or $T_{m}^{0}$ can lead to anomalies, as shown in the lower plots of Figs. 5-7.

\section{REFERENCES}

1. G.S.Y. Yeh and K.Z. Hong, PolymerEng. Sci., 19, 395(1979).

2. D. L. Krueger and G. S. Y. Yeh, J.Appl. Phys., 43, 4339(1972).

3. V. Tan and C. G. Gogos, Polymer Eng. Sci., 16, 512 (1976).

4. D. L. Krueger, Ph.D. Thesis, Univ. of Michigan (1972).

5. R. Ulrich and F. P. Price, J. Appl. Polym. Sci., 20, 1095(1976).

6. K. Z. Hong, unpublished data.

7. J. I. Lauritzen, Jr. and J. D. Hottman, J. Res. NBS, 64A, 73 (1960).

8. K. Z. Hong and G. S. Y. Yeh, "Strain-Induced Crystallization, Part V: Melting Behavior of Strain-Crystallized Polymers," in preparation.

9. L. Mandelkern, "Crystallization of Polymers," McGraw-Hill (1964). 